\title{
LA ACTIVIDAD FÍSICA EN LOS Y LAS JÓVENES MEXICANOS Y MEXICANAS: UN ANÁLISIS COMPARATIVO ENTRE LAS UNIVERSIDADES PÚBLICAS Y PRIVADAS.
}

\author{
Jaime García Rodríguez y Carlos Fonseca Hernández. \\ Universidad Autónoma del Estado de México. \\ jaimedrgarcia@prodigy.net.mx
}

\section{RESUMEN}

\begin{abstract}
La actividad física en la historia aparece desde el momento mismo en que el ser humano surge en la faz de la tierra como un personaje con necesidad de movimiento, el cual se ha manifestado desde el principio de la humanidad en varias facetas tales como el movimiento mecánico, laboral y social. Esto ha evolucionado directamente en proporción al desarrollo humano, llegando hasta nuestros días a presentarse como parte vital dentro de la formación de los individuos y como un medio indispensable para alcanzar salud; sin embargo, existe también un sesgo en la práctica de dicha actividad física y deportiva, que ha originado un culto al cuerpo basado en un posmodernismo influenciado por los medios masivos de comunicación y estándares de una supuesta belleza física en ocasiones inalcanzable a la realidad humana; lo que ha ocasionado la presencia de problemas físicos, biológicos, pero de igual manera psicológicos, que repercuten en la autoestima y autoimagen de la población joven y adolescente, que se encuentra en busca de su identidad. Los y las jóvenes actualmente se encuentran influenciados por un estilo de vida dominado por la modernidad y los avances tecnológicos, lo que deriva en una vida sedentaria que produce efectos nocivos en todos los sentidos y todos los ámbitos; lo cual aunado a programas de un sistema institucional educativo y deportivo establecido con innumerables carencias, nos lleva a complicar más la situación, donde se añaden factores perjudiciales que abarcan desde la ignorancia, la incapacidad e intereses personales, que no nos ayudan para edificar alternativas que comiencen a funcionar. Es primordial el establecer una solución pronta y efectiva encaminada en la sistematización de una actividad física adecuada basada en argumentos científicos y métodos acordes, que nos puedan proveer de los diversos beneficios que su práctica otorga y nos ayuden a detener el proceso de deterioro físico al cual el ritmo de vida actual no arroja.
\end{abstract}

PALABRAS CLAVES: Actividad Física, jóvenes, tecnología.

\section{ABSTRACT}

Physical activity appears in history from the moment when the human being comes in the face of the earth as a character inneed of movement, which has appeared since the beginning of mankind in various aspects such as mechanical motion, andsocial work. This has evolved directly in proportion to human development, reaching today to stand as a vital part of the training of individuals and as an indispensable means to achieve health, but there is also a bias in the practice of this activity and sport, which has created a cult of the body based on a postmodernism influenced by the mass media and standards of an alleged physical beauty sometimes unattainable human reality 
which has led to the presence of physical, biological, but equally psychological way, affecting self-esteem and selfimage of young people and adolescents, which is in search of his identity. These kids today are influenced by a lifestyle dominated by modernity and technological advances, resulting in a sedentary lifestyle which adversely in every way and all areas, which coupled with institutional programs educational system and countless sports established deficiencies, leads to further complicate the situation, where you add harmful factors ranging from ignorance, inability and personal interests, which do not help us to build alternatives to start working. It is essential to establish an early and effective aimed at systematizing adequate physical activity based on scientific arguments and methods in line, we can provide various benefits given practice and help us stop the process of physical deterioration which the pace of modern life does not shed.

KEYWORDS: physical activity, young, technology.

\section{INTRODUCCIÓN}

La preocupación por los estándares de belleza y un cuerpo estéticamente atractivo hacia los demás ha sido motivo de atención desde la antigüedad, la cual actualmente ha cobrado una fuerza mayor basándose en la influencia de una sociedad modernizada e influenciada por distintos medios de comunicación que intervienen de manera decisiva en la población y no necesariamente con bases científicas fundamentadas, provocando efectos secundarios nocivos en la salud de los individuos.

Ahora, igualmente es cada vez más frecuente y necesaria la práctica de ejercicio por los distintos grupos etáreos en nuestra comunidad, esto debido al inminente problema de obesidad y de enfermedades crónicas degenerativas que nos aquejan. Lo que al final de cuentas desencadena que se quiera realizar de la manera más rápida y con menos esfuerzo, como en la mayoría de nuestras metas como seres humanos, la ley del mínimo esfuerzo, generando a su vez de manera no deseada problemas de conducta entre algunas personas como la obsesión por alcanzar una mejor figura y por obtener mayor masa muscular.

En virtud de lo anterior, algunas personas acuden al uso y abuso de sustancias de tipo ergogénico para lograr objetivos deportivos que tienen que ver con la obtención de una mejor estética corporal y rendimiento físico de manera más pronta y supuestamente más efectiva; sin tomar en cuenta los posibles riesgos a la salud que puede implicar en algunas 


\section{MHSalud}

URL www.una.ac.cr/mhsalud

de las veces, generando problemas tales como los renales, hepáticos, dermatológicos, psicológicos, gastrointestinales, incluso llegando al cáncer.

Entre todos estos problemas causados, vale la pena mencionar los de conducta, como la dismorfia muscular, que es un trastorno que afecta de manera multidireccional al individuo y su entorno, que actualmente representa un área de oportunidad en nuestra sociedad.

En una sociedad posmoderna, cada vez más apegada a la tecnología en el uso de Internet, celulares, computadoras, videojuegos, redes sociales e incluso el sexo virtual; el sedentarismo se presenta cada vez con mayor frecuencia impactando en la salud de la población, siendo la principal causa de obesidad a nivel mundial, donde como país, ocupamos los primeros lugares, generando la aparición de enfermedades crónico degenerativas que nos aquejan con una frecuencia cada vez más evidente, ocasionando consecuencias a nivel económico, biológico y social en todos los sectores poblacionales.

Los y las jóvenes son un grupo etáreo vulnerable a los estilos de vida impuestos por los medios de comunicación e influenciables por la tecnología cada vez más avanzada que los conlleva a un modo de vida inactivo aplicando la ley del mínimo esfuerzo físico, condenando a esta población, que representa la fuerza económica futura del país, a padecer enfermedades propias de este sedentarismo y no sólo a eso, sino también a disminuir su ciclo de vida y de manera determinante la calidad de la misma.

Un ejemplo son nuestros(as) jóvenes de distintos centros universitarios de renombre como el Instituto Politécnico Nacional, La Universidad Autónoma del Estado de México, el Tecnológico de Monterrey, la Universidad del Valle de México, la Salle, la Universidad Justo Sierra y la Escuela Médico Naval; donde se practican un sinfín de disciplinas deportivas, pero que no son llevadas a cabo por el grueso de los alumnos en todos los casos, y como en todo en la vida, existe un área de oportunidad para mejorar ciertos aspectos de infraestructura y estrategia. 
Por eso, la importancia de introducir en esta población juvenil principalmente, un sistema de vida dinámico donde se lleve a cabo una actividad física adecuada, periódica, programada y bien prescrita, como una herramienta básica para conseguir y preservar la salud, ya que los innumerables beneficios fisiológicos, metabólicos, anatómicos, cognitivos, sociales y académicos que representa son inobjetables. Sin embargo, se debe tener mucho cuidado en no caer en excesos que nos pueden llevar a ocasionar trastornos de la autoimagen y autoestima; problemas que de igual manera se presentan primordialmente en adolescentes y jóvenes, debido a que son estos los que aún se encuentran en busca de una identidad y aceptación social. Se debe también, tomar en cuenta que diferentes disciplinas, entre ellas la nutrición, la psicología, la educación física y la medicina del deporte son factor determinante para que se pueda llevar a cabo una óptima práctica de la actividad física.

Así, el objeto de estudio del presente artículo es precisamente, realizar un comparativo entre la actividad física realizada en distintos centros universitarios, como un componente vital para el desarrollo integral de una sociedad posmoderna necesitada de mejorar su calidad y estilo de vida.

\section{DESARROLLO}

\section{LA TECNOLOGÍA, EL POSMODERNISMO Y LOS Y LAS JÓVENES}

En la actualidad, no se pone en duda la importancia que tienen los y las jóvenes para el desarrollo de un país, de hecho se les considera como actores estratégicos para el futuro de México, sin embargo, a pesar de ello han sido poco estudiados, atendiendo a la suposición de que son una población sana, y en periodos anteriores a la década de los sesenta pasaron inadvertidos a todo el proceso de modernización e industrialización del México contemporáneo. De igual manera, es indiscutible que la tecnología es la generadora de grandes cambios sociales y culturales a los cuales los individuos se han adaptado en las relaciones laborales, sociales y familiares (Blanco, 2006). 
Ya no es posible pensar en el desarrollo humano sin tomar en cuenta el uso de los medios tecnológicos, pues se han convertido en la vía por la cual el ser humano reemplaza su medio natural por un ambiente técnico, fenómeno observado principalmente a finales del siglo XIX y finales del XX; es decir que para las personas, el entorno toma otro significado y un valor drásticamente diferente, lo natural ya no parece tan atractivo como lo tecnológico, especialmente para las personas jóvenes (Blanco, 2006).

La tecnología se ha convertido en la única forma moderna de producir aparatos útiles y valiosos para las exigencias nuestra sociedad, además de dominar la mayoría de las actividades humanas. Al tener en cuenta el impacto que la tecnología posee para la sociedad y su cultura, surge la inquietud de saber de manera más precisa cuáles son las consecuencias de esta influencia y cual puede ser su alcance, así como que tanto se puede controlar o disminuir dicho flujo tecnológico (Blanco, 2006).

El desarrollo de las nuevas tecnologías electrónicas para la transmisión y almacenamiento de datos, o simplemente para ofrecer mayores opciones de esparcimiento, comunicación y aprendizaje, forman parte de los procesos más complejos y novedosos en nuestra sociedad. Los medios de comunicación se han constituido en un ambiente donde se desenvuelve nuestra vida, donde se crean y reproducen lenguajes, conocimientos, valores comportamientos, actitudes y orientaciones sociales.

Según Quevedo (2003) la aceleración tecnológica modificó también de manera profunda y desigual el perfil de las sociedades de fin de siglo, la constitución del espacio público y los modos de vida de sus integrantes, al tiempo que cambió los referentes culturales en el caso de los y las jóvenes. Debido a diversas causas se puede afirmar que la televisión ha colonizado el tiempo libre de la gente, y esta población es consumidora intensiva de tecnologías de comunicación: videos, cine, radio, videojuegos; y en los sectores sociales más altos, las tecnologías ligadas a la computación: chat, internet, e-mail, etc. 
Las personas se pueden interrelacionar a través de las redes sociales; de hecho con tal importancia, que según registros de google México, mientras la radio tardó 38 años en llegar a 50 millones de usuarios en el mundo, y la televisión se tomó 12 en conseguir un igual número de televidentes, el Internet alcanzó 50 millones de internautas en 4 años en 1995, y para 2010 se contabilizaron más de 1900 millones de usuarios a seca mundial, cifra que el día hoy seguramente ya está rebasada (García, 2011).

Esta presencia de la tecnología en la vida cotidiana se ha transformado en un problema central en la educación, pues constituye hoy, un agente de socialización tan importante como la escuela o la familia, sólo que con diversas directrices que no necesariamente nos llevan a un resultado benéfico (Quevedo, 2003).

El peso específico que tiene la tecnología en la funcionalidad integral de la sociedad, recae directamente en el grupo poblacional de jóvenes y adolescentes, por que se encuentran en una etapa de formación en todos los sentidos y resultan completamente influenciables por un estilo de vida moderno y tecnológico, donde el valor personal es proporcional al tipo de tecnología utilizada de forma individual como lo son los celulares, las computadoras portátiles, ipod, ipad, iphone, televisión de paga, Internet, etc.

Y no sólo es la influencia de la tecnología, sino también el estilo personal, marcado por cánones de belleza y actualidad impuestos por los medios de comunicación, dirigidos a obtener determinada silueta corporal, autos, ropa y accesorios en muchas de las ocasiones inalcanzables para la realidad de los individuos; algunas veces por causas sociales como el propio nivel socioeconómico, y otras de tipo biológico como por ejemplo un somatotipo o carga genética que no les permite tener un cuerpo tan adelgazado, que es como lo marca la moda actual.

Como los y las jóvenes representan la esperanza futura, debemos hacer énfasis en su desarrollo integral como individuos, que parte de un entorno social ávido de un cambio en los estilos de vida que marcan un camino hasta ahora directo a una vida sedentaria, llena de 
comodidades que conllevan a inactividad, teniendo diversas complicaciones no sólo en el aspecto biológico, sino social, cultural y económico.

Se debe de aprovechar la tecnología existente hasta el momento, pero sin abusar del ahorro de energía que produce la inactividad física, sobre todo en este grupo etáreo, pero sin descuidar que los demás grupos poblacionales no se encuentran exentos de padecer el mismo problema. Un ejemplo claro son los videojuegos, que han venido a dejar obsoletas las tradicionales recreaciones basadas en movimientos y actividad continua; y aunque algunos de ellos requieren de cierto movimiento para que se lleven a cabo, como el caso del Wii, videojuego que cuenta con un controlador que incorpora sensores de movimiento que le permiten tres ejes de libertad, y que el usuario ejecuta a través de mecánica corporal; definitivamente no reemplazan los beneficios que brinda la actividad física practicada de manera tradicional. De igual manera, herramientas básicas como el Internet deben ser usadas con fines objetivos y moderación, evitando pasar la mayor parte del tiempo sentado ante una computadora interactuando en base a redes sociales establecidas por este medio, y que en muchas de las ocasiones sólo representan una pérdida de tiempo y a veces hasta un peligro inminente de poder ser víctima de la delincuencia existente a través de esta vía.

Todos estos medios de comunicación y tecnología están cada vez más al alcance de la mano de los diversos estratos sociales, obviamente siendo más accesibles para los jóvenes de nivel socioeconómico alto, pero que no exime de su uso a los demás niveles. Si realizáramos un comparativo de jóvenes que acuden a universidades privadas con respecto a jóvenes pertenecientes a universidades públicas, se podría decir que ambos se encuentran igualmente influenciados por los aparatos tecnológicos, sin embargo, probablemente la única diferencia es la forma en que los consiguen o los usan unos y otros. 


\section{MHSalud}

URL $\underline{\text { www.una.ac.cr/mhsalud }}$

\section{Imagen 1.}

Clase de stretching en alumnos de la licenciatura de nutrición en la Universidad Justo Sierra.

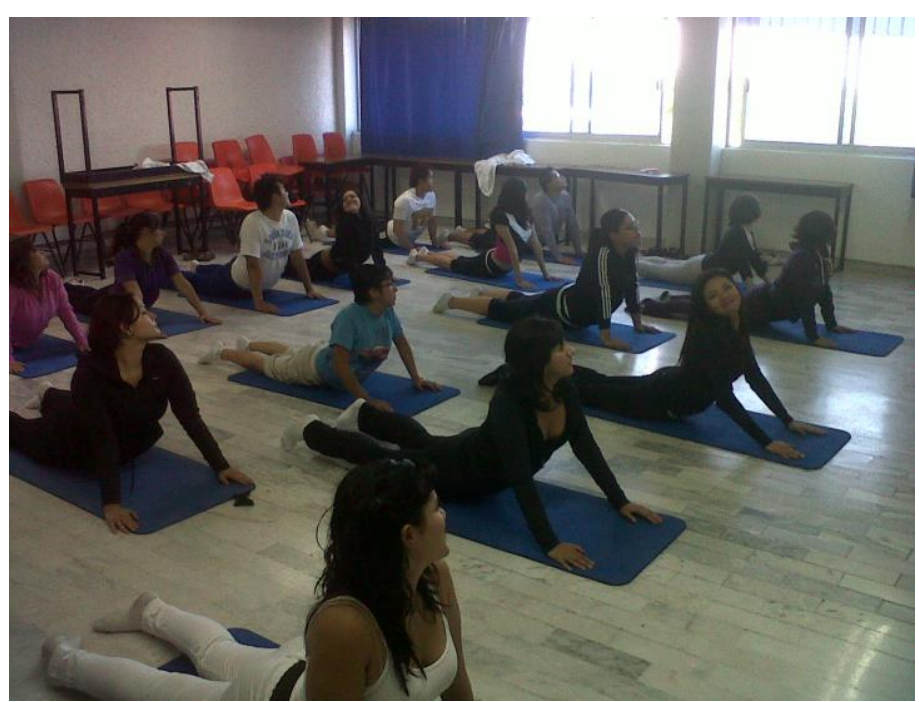

Fuente: Fotografía tomada de archivo personal, se cuenta con todos los derechos para su uso en este artículo

\section{JÓVENES SEDENTARIOS, FUTUROS ADULTOS ENFERMOS, UN PROBLEMA} PARA EL PAÍS.

La necesidad de movimiento en el desarrollo de las actividades humanas cotidianas se ha reducido bajo los efectos de la modernización y la automatización, los cuales han generado cambios en el estilo de vida de las poblaciones, dicha tendencia ha aumentado de forma acelerada y se ha establecido como la conceptualización de sedentarismo; el cual fue calificado por la Organización Mundial de la Salud (OMS) y la Organización Panamericana de la Salud (OPS) en el 2003, como un factor de riesgo para la aparición de enfermedades crónicas no transmisibles de la edad adulta, y que exhibe entre sus rasgos fundamentales la presencia de hipocinetismo, es decir poco movimiento, condición que se manifiesta por el predominio de un nivel de actividad física insuficiente para lograr un estado de salud general recomendable (Moya, García, Lucena, Casañas, Brito, Rodríguez, Flores y Cordero, 2006). 
Debemos partir entonces, que la salud no es algo estático sino un proceso dinámico donde, tanto el individuo como su entorno tienen el poder de cambiar su situación y dirigirla hacia un mejor nivel y calidad de vida; de la misma manera entonces, una de las estrategias para conseguirlo es también dinámica: la adecuada práctica de actividad física; que hasta hoy, debido a diversas circunstancias no se lleva a cabo, y menos de la manera esperada para poder conseguir los beneficios que en la salud otorga; a pesar de que supuestamente la población se encuentra en el entendido de que el practicar ejercicio contrae un mejor estado de salud.

En todo el mundo existe una creciente preocupación por las consecuencias que provoca la inactividad física; ya que el sedentarismo causa diversos factores adversos en el individuo como: el aumento del peso corporal, disminución de la flexibilidad, hipotrofia muscular, disminución de los tiempos de reacción, mala circulación, mala postura, dorsalgias y lumbalgias, aparición de enfermedades crónico degenerativas, cansancio crónico, baja autoestima y poca interrelación social (Ramírez, Vinaccia y Suárez, 2004).

Recientes estudios demuestran al sedentarismo como un factor desencadenante de un número importante de enfermedades crónico degenerativas como la hipertensión arterial, la diabetes, y la obesidad entre otras. En Latinoamérica se hallan cifras que estiman que más de un cuarto de la población mayor a 14 años no practica una actividad física o deporte. Este estilo de vida sedentario no sólo atenta contra la calidad de vida de la población, provocando la aparición de enfermedades, sino que además, tiene un alto costo económico para el país. Se estima que un $20 \%$ del presupuesto destinado a las instituciones de salud, podría ahorrarse si se aplicaran programas y proyectos que favorezcan el desarrollo de actividad física y deporte. Así, los recursos serían orientados de manera eficiente hacia planes de prevención y mejoramiento de la salud pública y no solamente a cubrir las necesidades y exigencias que ocasionan las enfermedades (Ramírez et al., 2004).

No podemos olvidar que en la actualidad nuestro país ocupa los primeros lugares en obesidad, principalmente a nivel infantil, provocado precisamente por este estilo de vida sedentario, debido a la falta de promoción de la actividad física en todos los sectores de la 
comunidad por parte de las instituciones de salud y deportivas, la falta de profesionales de la salud capacitados en el tema, programas de activación física francamente no diseñados con bases científicas o realistas acorde a nuestra población, además de una sociedad cada vez más apegada a la tecnología.

La obesidad y el sobrepeso son uno de los principales problemas de Salud Pública, según la OMS, la obesidad es la enfermedad metabólica más frecuente en los países desarrollados y se considera como una epidemia (Who, 1998). La prevención es el tratamiento ideal, y la escuela es el lugar ideal para educar sobre hábitos alimenticios saludables, así como para promover y facilitar el desarrollo del ejercicio físico. Los centros escolares juegan un importante papel en la promoción de la salud debido al carácter obligatorio de la educación para toda la población a nivel básico, su naturaleza de espacio educativo y la cantidad de tiempo que permanecen ahí los alumnos (Santos, 2005).

Sin embargo, aún es distante el poder lograr este nivel de educación en nuestros (as) jóvenes; según la revisión realizada por Simons-Morton y Parcel (1988) sobre los patrones de actividad física de los y las jóvenes devela un gran problema: la sociedad no ha sabido persuadir a la gente joven para que adopte un estilo de vida activo y se implique en la práctica frecuente de ejercicio físico. En este sentido, se debe tomar en cuenta que la etapa escolar es fundamental para fomentar un estilo de vida activo, teniendo en cuenta que se trata de un periodo en el que se necesitan aprender habilidades y conocimiento práctico que influirá y facilitará el compromiso con una vida activa (Devís y Peiro, 1997).

Hay que tomar en cuenta que el esfuerzo que se realice en los sistemas escolares tampoco será suficiente si en el núcleo principal de la sociedad, que es la familia, no se aplican estrategias específicas para promover el estilo de vida activo. Los programas educativos en este sentido deben ir dirigidos paralelamente a las dos entidades: la escuela y la familia. Pero, la realidad es que ambos sectores se encuentran completamente olvidados, y cuando se pretende aplicar programas para promover la actividad física, estos resultan insuficientes y obsoletos. Al paso que vamos hasta el momento, todos estos y estas jóvenes 
sedentarios(as) de hoy, serán los adultos (as) mayores (predominantes en la pirámide poblacional futura) enfermos de mañana, afectando no sólo su individualidad y su núcleo familiar, sino al equilibrio económico del país, incrementando el gasto público en servicios médicos, medicinas, incapacidades y un sistema de pensiones de por sí ya desgastado.

\section{BENEFICIOS DE PRACTICAR ACTIVIDAD FÍSICA ADECUADAMENTE.}

El término actividad física hace referencia a cualquier movimiento corporal producido por los músculos esqueléticos y que tiene como resultado un gasto energético que se añade al metabolismo basal. En los últimos años se ha profundizado cada vez más en el estudio de la actividad física, tanto en los efectos saludables de su práctica habitual como en la relación que su ausencia mantiene con el desarrollo, mantenimiento y agravamiento de diversas enfermedades crónicas. El ejercicio es un tipo de actividad física, pero con un sistema, un método, practicado de manera regular y que puede estar reglado o no (Varo, Martínez y Martínez, 2003).

En los últimos años, estudios científicos han demostrado los beneficios producidos en la salud por la práctica de actividad física regular, y como consecuencia la promoción de la actividad física está siendo reconocida como una cuestión a tratar por la salud pública (Pate, 1995).

En el ejercicio físico adecuado e individualizado, realizándolo con frecuencia, en forma continua y progresiva, queda comprobado científicamente que: existe un desarrollo y crecimiento equilibrado y armónico, mantiene el funcionamiento óptimo de los aparatos y sistemas del organismo primordialmente el cardiovascular y músculo esquelético, reduce el riesgo de enfermedades crónico degenerativas, mejora el control de las mismas, contribuye al bienestar mental y psicológico, mejora el estado de ánimo, mejora la autoestima y da una consideración positiva sobre la imagen corporal (Devís y Peiro, 1997); tomando en cuenta lo importante que resulta mantener una adecuada autoestima y autoimagen en los y las jóvenes principalmente, para lograr un desarrollo integral que garantice la funcionalidad e integración social de estos individuos. Así también, Paffenbarger, Hyde, Wing, Lee, Jung y 
Kampert, (1993) demostraron que el incremento en los niveles de actividad física ha sido eficaz para aumentar la longevidad: las personas físicamente activas viven alrededor de 2 años más que las inactivas; aunque independientemente de poder alcanzar o no un mayor número de años al ciclo de vida, lo más importante será la calidad que se le pueda agregar a estos años (Ramírez et al., 2004).

Entre otros beneficios, algunas investigaciones realizadas demuestran el fuerte impacto que tiene la actividad física sobre la cognición, la socialización y el rendimiento académico. Estudios desarrollados por la universidad de Illinois, en los Estados Unidos, determinaron que a mayor actividad aeróbica, menor degeneración neuronal. Un trabajo realizado por el doctor Kubota de la Universidad de Handa (Japón), demostró con jóvenes que realizaron actividad física por 30 minutos tres veces por semana durante tres meses, obtener una mejor función del lóbulo frontal del cerebro, comprobado a través de pruebas de inteligencia específicas. Se encontró una relación positiva entre la actividad física y el rendimiento académico en estudios realizados por el Departamento de Educación del estado de California en los Estados Unidos, que apoyan la idea de que dedicar un tiempo sustancial a la actividad física en las escuelas, puede traer beneficios en el rendimiento académico de los niños (Ramírez et al., 2004).

Carratala y Carratala (1999) mostraron que se atribuye al deporte el poder magnificar valores psicosociales como el respeto por el otro, la responsabilidad y el compañerismo. Gutiérrez (1995), concluye que los valores sociales y personales son los más propicios de alcanzar a través de la actividad física y el deporte.

Para poder alcanzar los beneficios que la actividad física provee, es importante mencionar que se debe llevar a cabo de manera adecuada, con metas individualizadas y alcanzables, adaptada al tipo de población a la que va dirigida, reduciendo al máximo los riesgos de su práctica, reuniendo todos los implementos necesarios en cuanto a indumentaria, recursos materiales deportivos, planes de entrenamiento específicos, profesionales en la preparación física para prescribir los planes de entrenamiento, una nutrición equilibrada y auxiliarse de 
una especialidad médica como la Medicina del Deporte, que maximice las posibilidades de éxito en los objetivos marcados y se minimicen los accidentes propios de dicha actividad. Lo cual, lamentablemente no se lleva a cabo en la mayoría de las ocasiones; donde se aplican programas de actividad física generalizados, sin evaluaciones previas ni valoraciones periódicas, que se realizan en base a políticas no pensadas para las necesidades reales de la población.

Es de subrayar la relevancia de la actividad física como un potencial indiscutible para el desarrollo individual y colectivo; y por tanto, fundamental considerarla como un pilar indiscutible dentro del rubro de la salud. Además, los beneficios no son meramente físicos o estéticos, las ventajas a nivel cognitivo y social, sin dejar a un lado el aprovechamiento escolar, representan un avance evolutivo en las tres esferas del individuo como unidad biopsicosocial.

Queda claro que es primordial promover y aplicar programas de activación en nuestro grupo etáreo más vulnerable que son los y las niños (as) y jóvenes; sin pasar por alto que los beneficios que la actividad física brinda son en general para todas las edades. Solo hay que tomar en cuenta que dicha actividad o ejercicio debe ser específico acorde a edad, sexo, condiciones ambientales, estado de salud, condición física, etapa de entrenamiento, metas y objetivos individuales. Y sobre todo, esta actividad debe ser evaluada, vigilada y prescrita de manera multidisciplinaria por diferentes áreas: Medicina del Deporte, nutrición, psicología, kinesiología, terapia física, y la metodología del entrenamiento, quienes trabajarán en conjunto para tener éxito en esta ardua tarea de la promoción de la salud y la mejora de los estilos de vida en niños y jóvenes a través de la actividad física.

Desafortunadamente, la mayor parte de la población en nuestro país no hace ejercicio, desconoce los beneficios que este puede aportarle, e ignora la manera apropiada para llevarlo a cabo; y quien llega a practicarlo, generalmente lo hace de manera empírica y desorientada, influenciado por lo medios de comunicación y personas no capacitadas ni expertas en el ámbito deportivo, como para dar recomendaciones o aplicar programas de 


\section{MHSalud}

URL $\underline{\text { www.una.ac.cr/mhsalud }}$

entrenamiento. Por tanto, urge unir esfuerzos en todas las disciplinas de la salud para establecer estrategias de activación y educación sobre la actividad física, que cubran las expectativas y necesidades que la población demanda.

Queda el reto para realizar investigación en el área, elaborando un programa de promoción de la actividad física aplicable a nuestra población, que evalúe el incremento de actividad física de la comunidad, y el resultado en calidad de vida relacionada con salud y forma física. Como lo realizado en el protocolo para la evaluación multicéntrica del programa experimental de promoción de la actividad física (Grandes, Sánchez y Torcal, 2003).

\section{Imagen 2.}

Clase de stretching en alumnos de la maestría en fisioterapia deportiva en la Universidad del Valle de México, Campus Coyoacán.

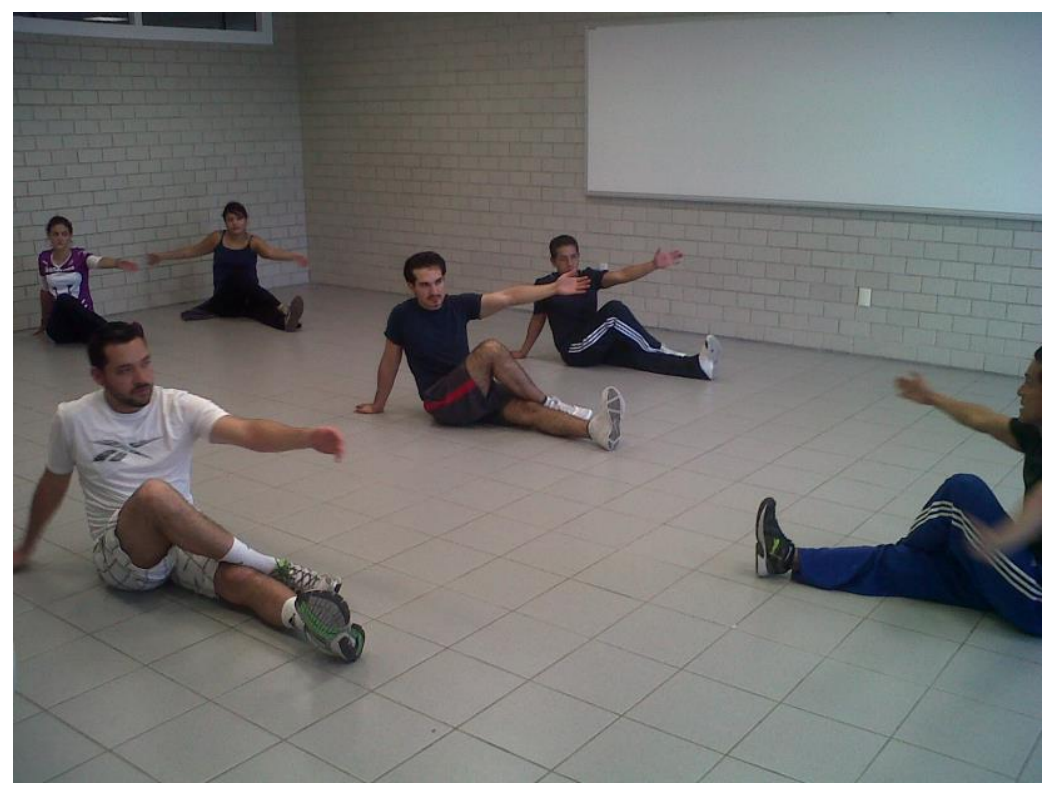

Fuente: Fotografía tomada de archivo personal, se cuenta con todos los derechos para su uso en este artículo

\section{LA ACTIVIDAD FÍSICA EN MÉXICO.}

Actualmente en nuestro país existe una reforma a los artículos 65 y 66 de la Ley General de Salud donde se instruye a las autoridades sanitarias y educativas a promover la obligatoriedad escolar de practicar 30 minutos de ejercicio físico para prevenir 
padecimientos de sobrepeso y obesidad que ponen en peligro la salud física y mental de los y las menores, aunque en la realidad esto no se lleve a cabo ni en tiempo ni en forma, resultando insuficiente y mal aplicado. Así, con 372 votos a favor en el pleno de la cámara de diputados, se dio luz verde al dictamen de la Comisión de Salud, en el que se advierte la necesidad de combatir la obesidad que actualmente padece el 30 por ciento de la población escolar del país. De igual manera se aprobó la reforma para regular la venta de alimentos chatarra en las escuelas como otra medida para solucionar el problema.

Existen también, programas regionales y nacionales de activación física escolar en el nivel educativo básico, que es donde se encuentra el grupo etáreo de mayor problema. Sin embargo, la realidad es que hasta este momento no han funcionado de la manera esperada ninguno de estos programas, y es probablemente por que se basan como la mayoría de las políticas públicas en nuestro país, sólo en buenas intenciones y atendiendo a los intereses personales o de grupos específicos, sin tomar en cuenta aspectos regionales, individuales, sin planes de control y seguimiento; y donde la mayoría de las veces, los encargados de aplicar dichos programas y protocolos de activación física no tienen la formación profesional ni la información necesaria para aplicar de manera eficiente la encomienda, y en muchos de los casos, el plan de activación física y de alimentación es tomado como una mera norma que hay que cumplir al vapor sin la conciencia de la necesidad real de solucionar el problema tan grave que nos aqueja.

En el nivel educativo superior la situación tampoco es tan alentadora, pues pocas son las universidades de nuestro país, que realmente le dan la importancia merecida a la actividad física y deportiva, donde realmente no toman en cuenta los beneficios formativos que puede otorgar, es tomada como un aspecto extra académico de menor nivel de jerarquía en el currículo escolar; y lo que es peor, en muchas ocasiones es practicada e impartida por personal no calificado o experto en el área, basándose solamente en la propia experiencia deportiva y en el empirismo puro, sin pensar que la educación física es un constructo que abarca diversas disciplinas de la ciencia para establecer sus bases metodológicas y fisiológicas, que debe de practicarse de manera científica y multidisciplinaria. Hacen falta 


\section{MHSalud}

URL www.una.ac.cr/mhsalud

recursos materiales, infraestructura y personal capacitado que garantice un buen nivel de desarrollo de la actividad en estos niveles educativos.

El deporte a nivel privado en los gimnasios y clubes deportivos cuenta con las mismas o peores carencias en cuanto a los recursos y los métodos de entrenamiento aplicados a los usuarios, pero con un elemento más en contra: el uso de sustancias ergogénicas, que dependiendo su origen, como en el caso de los anabólicos, pueden causar efectos devastadores a mediano y largo plazo en las personas que los utilizan; y como hablamos de productos que son introducidos como una alternativa más rápida para obtener resultados sobre todo estéticos, resultan un potencial muy atractivo, sobre todo para la población joven que resulta, como siempre, la más susceptible.

Y por último, pero no así en orden de importancia, el alto rendimiento; donde tampoco es la excepción, pues la falta de recursos, becas deportivas, apoyos a talentos deportivos, falta de profesionales de apoyo como médicos especialistas en Medicina del Deporte, psicólogos, nutriólogos, terapistas, metodólogos; una escasa evaluación morfofuncional previa y de seguimiento a los deportistas, e intereses personales que se sobreponen al bien común, afectan y limitan el desarrollo del deporte profesional en México.

\section{Imagen 3.}

Sesión de recuperación con electroterapia en competidores de una carrera atlética celebrada en el Tecnológico de Monterrey Campus Santa Fe.

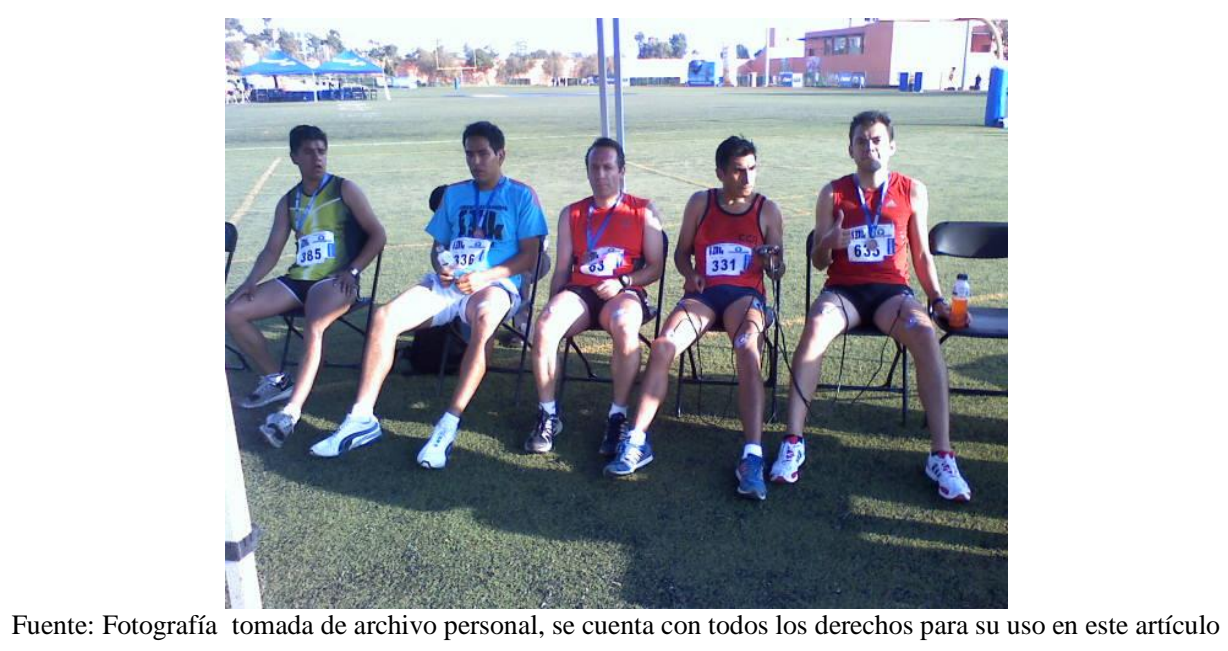




\section{LA ACTIVIDAD FÍSICA Y LA MEDICINA DEL DEPORTE EN ALGUNOS CENTROS} UNIVERSITARIOS EN MÉXICO: UNA REVISIÓN DE LOS CASOS EN LA UNIVERSIDAD AUTÓNOMA DEL ESTADO DE MÉXICO, INSTITUTO POLITÉCNICO NACIONAL, EL INSTITUTO TECNOLÓGICO Y DE ESTUDIOS SUPERIORES DE MONTERREY, LA UNIVERSIDAD DEL VALLE DE MÉXICO, LA SALLE , JUSTO SIERRA, Y LA ESCUELA MÉDICO NAVAL.

La actividad física y el deporte son una actividad primordial de carácter extraacadémico que se lleva a cabo en los distintos centro universitarios en nuestro país; pero que sin embargo, no se le da la importancia que representa dentro del desarrollo integral de los y las jóvenes universitarios(as), por distintas razones o impedimentos, pero que al final de cuentas es un área a la que se le da un peso secundario, sin tomar en cuenta los beneficios y efectos positivos que arroja para esta población.

Así también, hay que darle una mención específica a un área necesaria para desarrollar dicha actividad física dentro de las instalaciones universitarias, y esta es la medicina del deporte. La medicina del deporte es una especialidad médica desafortunadamente poco conocida en México, debido al poco número de especialistas que existen en el país; sin embargo es una disciplina fundamental para que se lleve a cabo una adecuada y bien prescrita actividad físico deportiva, no sólo los deportistas de alto rendimiento, sino en todo aquel que practique algún tipo de actividad física. Es una disciplina que se encarga de la prevención y atención de lesiones del sistema músculo esquelético, la evaluación previa la actividad deportiva a través de evaluaciones morfofuncionales, corrección de la postura y biomecánica, detección de talentos deportivos, eficiencia la adecuación física ayudando a mejorar el rendimiento físico de los individuos, e interrelaciona con otras disciplinas para mejorar los planes de entrenamiento, recomendaciones nutricionales, y rehabilitación de lesiones provocadas por el deporte. 
La medicina del deporte en nuestro medio se desenvuelve a nivel de la medicina privada, instituciones deportivas y algunas instituciones educativas, y aunque parezca irónico, en el sector salud no está lo suficientemente diseminada a pesar de las necesidades que existen en dicha área con respecto a contar con expertos en este rubro, que ayuden a la atención y prevención de los problemas de obesidad y enfermedades crónico-degenerativas que nos persiguen de manera amenazante cada vez con más fuerza.

Ahora, hablando ya específicamente de las escuelas, un rubro primordial en el desarrollo poblacional, centro fundamental de influencia en nuestros(as) jóvenes, y objetivo primario de este análisis; se expondrá un punto de vista personal y profesional del trabajo que se lleva a cabo desde el aspecto deportivo en algunas de ellas.

Para ello habrá que ejemplificar algunas instituciones universitarias públicas y privadas donde la práctica de actividad física y de la medicina deportiva están presentes; tal es el caso del Instituto Tecnológico y de Estudios Superiores de Monterrey, el Instituto Politécnico Nacional, la Universidad Autónoma del Estado de México, la Universidad del Valle de México, La Salle, la Universidad Justo Sierra y la Escuela Médico Naval; donde según la experiencia personal, se llevan a cabo diversas disciplinas deportivas, competencias locales, universiadas e intercampus, de donde surgen algunos talentos deportivos que llevan a cabo programas de entrenamiento especializados, en la mayoría de los casos con instalaciones adecuadas y de primer nivel. Sin embargo, existe aún una amplia área de oportunidad, donde se pueden mejorar los comedores para los deportistas, la infraestructura, el mantenimiento, los apoyos y becas para los alumnos, las áreas destinadas para la rehabilitación y terapia física, y sobre todo los recursos humanos especializados, ya que en algunas ocasiones son pocos en número, y en otras hace falta que realmente sean profesionales certificados en su área.

En las universidades públicas, el tipo de población, es tendiente a practicar la actividad física con probablemente menos recursos y apoyos económicos, sin embargo existe la infraestructura suficiente, aunque sea sólo a nivel central, no así en las unidades académicas 
descentralizadas, como en el caso de la Universidad Autónoma del Estado de México; como para que, con ayuda de profesionales de la misma institución, se puedan llevar a cabo programas eficientes y evaluables en rendimiento físico deportivo; sin embargo hace falta mucho por hacer en el área, y aplicarse en detección de talentos deportivos, ya que la fuga de estos a instituciones privadas, debido a las becas y apoyos que se les ofrece, es algo muy frecuente y que al final perjudica a estas universidades.

En las universidades privadas se ofrecen becas deportivas e instalaciones con alta tecnología, en la mayoría de los casos, para la práctica de las diversas disciplinas deportivas, con un sinfín de oferta en actividades en todas las áreas; sin embargo, a pesar de ello, también probablemente debido al tipo de población, no hay una cobertura total en la práctica de las mismas; aunque puede ser que si sea fundamental la cuestión económica, para que los alumnos se integren a las actividades deportivas, pues consideramos que no es lo mismo tener actividades académicas y deportivas como parte de un día normal, que añadirle actividades laborales que se tengan que desempeñar por necesidad económica en algunos casos de los alumnos de universidades públicas. Aunque no es una limitante determinante para no realizar actividad, ya que con una adecuada administración y una calendarización oportuna se puede hacer eficiente el tiempo y lograr el objetivo.

Con respecto a la inclusión de la medicina del deporte en estos centros educativos, la mayor parte le dan cierta importancia, pero faltando aún promoverla y apoyarla de manera más incisiva, sobre todo en los recursos materiales e infraestructura apropiada; y en la medida que esto suceda, los resultados en las competencias deportivas, el menor número de lesionados y mejor control de los deportistas, se hará evidente.

En la tabla 1 se exponen los principales aspectos que se diferencian acorde a las características particulares de las diversas universidades. 


\section{MHSalud \\ Revista en Ciencias del Movimiento Humano y Sal}

URL $\underline{\text { www.una.ac.cr/mhsalud }}$

\section{Tabla 1.}

Aspectos diferenciales en recursos humanos e infraestructura deportiva en distintas universidades.

\begin{tabular}{|c|c|c|c|c|c|}
\hline Universidad & $\begin{array}{c}\text { Médicos } \\
\text { especializados }\end{array}$ & $\begin{array}{c}\text { Infraestructura } \\
\text { deportiva }\end{array}$ & $\begin{array}{l}\text { Entrenadores } \\
\text { especializados }\end{array}$ & $\begin{array}{c}\text { Diversidad en las } \\
\text { disciplinas } \\
\text { deportivas }\end{array}$ & $\begin{array}{c}\text { Laboratorio o } \\
\text { Área de Medicina } \\
\text { del Deporte }\end{array}$ \\
\hline Tec de Monterrey Campus Santa Fe & $\mathrm{Si}$ & $\mathrm{Si}$ & $\mathrm{Si}$ & $\mathrm{Si}$ & $\mathrm{Si}$ \\
\hline UVM Coyoacán & No & $\mathrm{Si}$ & $\mathrm{Si}$ & $\mathrm{Si}$ & No \\
\hline Justo Sierra Acueducto & No & $\mathrm{Si}$ & $\mathrm{Si}$ & $\mathrm{Si}$ & No \\
\hline Salle Neza & $\mathrm{Si}$ & $\mathrm{Si}$ & $\mathrm{Si}$ & $\mathrm{Si}$ & Aún no \\
\hline IPN ESM & $\mathrm{Si}$ & Pocas & No & $\mathrm{Si}$ & $\mathrm{Si}$ \\
\hline Escuela Médico Naval & No & Pocas & No & No & No \\
\hline UAEM UAP Neza & $\mathrm{Si}$ & Pocas & $\mathrm{Si}$ & $\mathrm{Si}$ & $\mathrm{Si}$ \\
\hline
\end{tabular}

Fuente: Elaboración propia.

\section{Imagen 4.}

Sesión de TRX en alumnos de la Escuela Médico Naval

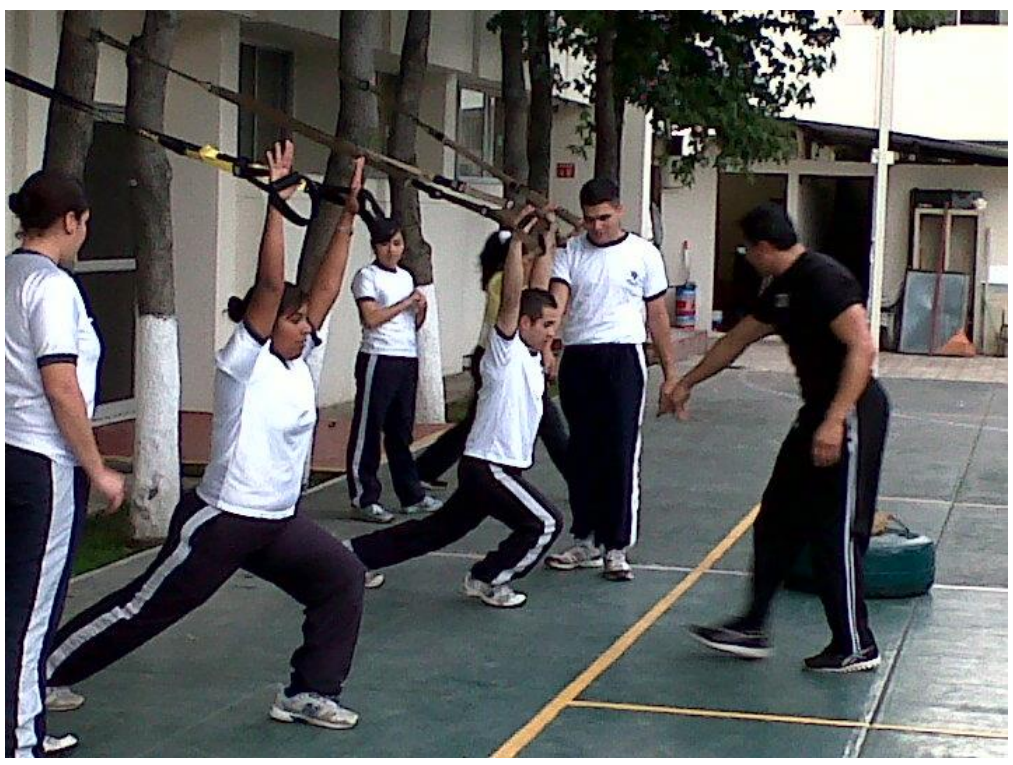

Fuente: Fotografía tomada de archivo personal, se cuenta con todos los derechos para su uso en este artículo 


\section{MHSalud \\ Revista en Ciencias del Movimiento Humano y Sa}

URL $\underline{\text { www.una.ac.cr/mhsalud }}$

\section{Imagen 5.}

Sesión de TRX en alumnos de la Escuela Médico Naval.

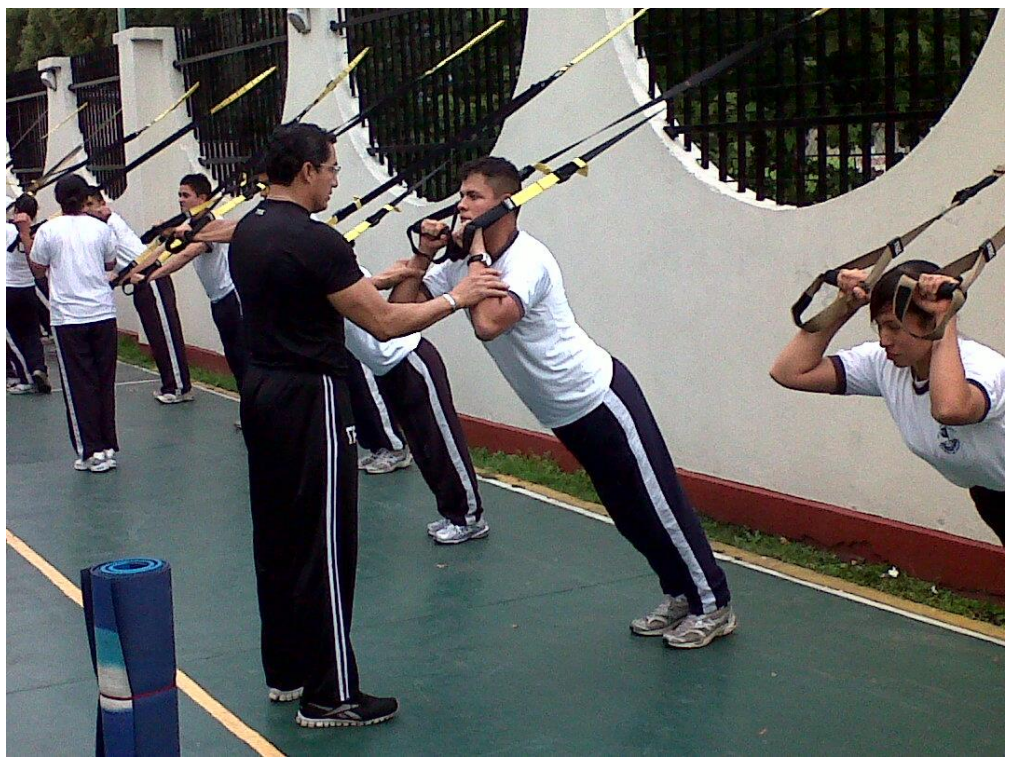

Fuente: Fotografía tomada de archivo personal, se cuenta con todos los derechos para su uso en este artículo

\section{Imagen 6.}

Sesión de TRX en alumnos de posgrado de medicina del deporte del IPN.

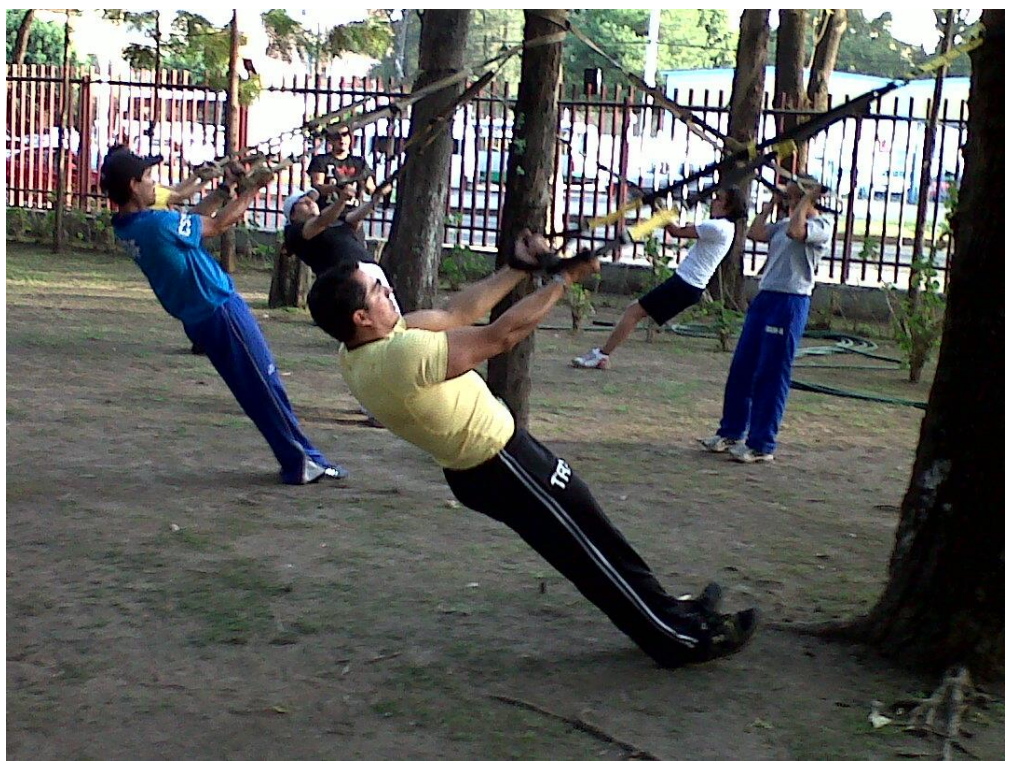

Fuente: Fotografía tomada de archivo personal, se cuenta con todos los derechos para su uso en este artículo 


\section{POSIBLES ALTERNATIVAS}

Habrá que realizar una revisión exhaustiva de los programas de activación física existentes en el país hasta el momento, dirigidos a grupos poblacionales diferentes, tomado en cuenta las particularidades regionales precisamente de esas poblaciones, así como los recursos humanos y materiales para llevarlos a cabo; establecer nuevos programas y proyectos que favorezcan el desarrollo de la actividad física y una buena alimentación, en base a la investigación a través de estudios regionales sobre hábitos alimentarios, actividades realizadas y recursos posibles de una población específica, que en este caso y en orden de importancia, es la integrada por nuestros(as) jóvenes.

Eliminar la intromisión de las empresas de productos alimentarios en las políticas y estatutos que se establezcan en las leyes y normas para la venta e introducción de alimentos en las escuelas, para evitar el sesgo hacia los intereses particulares de las mismas; así como, determinar el tipo de alimentos que se pondrán a la venta en las escuelas, como los alimentos llamados chatarra, como las frituras, golosinas y refrescos, que desafortunadamente aún se siguen ofertando en la mayoría de las escuelas, supuestamente cuidando la ración, cuestión realmente inefectiva ya que el reducir la cantidad en las porciones de los productos no es la solución, pues nadie regula que se venda más de una porción al mismo alumno, y al final de cuentas es importante la calidad y la cantidad de los alimentos.

El promocionar por todos los medios posibles en todos los sectores de la sociedad, la importancia de la práctica de actividad física adecuada e individualizada, que sea prescrita por personas capacitadas y no se realice de forma empírica; darle el peso específico que tiene en todos los lugares donde se imparte, tanto a nivel escolar, recreativo, privado o de alto rendimiento.

Contratar a profesionales de la salud especialistas en el área, tanto para diseñar los programas y estrategias, como para la aplicación de los mismos, que intervengan en las 
diversas instituciones tanto de educación y salud, como las dedicadas a la disciplina deportiva. Hasta hoy, la actividad física a nivel institucional y privado se encuentra no especializada, pues no basa su desarrollo en el apoyo de los expertos de las diferentes áreas de la salud, necesarios para llevar a cabo una actividad física plena y funcional.

El gobierno debe transformar esta situación, trabajando conjuntamente con los profesionales de la salud especialistas en el área, de una manera multidisciplinaria e interdisciplinaria para establecer las posibles estrategias, soluciones, políticas públicas y planes de intervención. Hace falta abrir un camino laboral en las instituciones de educación, a profesionales de la salud como el educador para la salud, el nutriólogo, el psicólogo y el médico del deporte, para que se apliquen los programas establecidos de manera específica y se realicen las evaluaciones previas y adecuadas a los escolares. Dentro de las instituciones deportivas del país existe carencia de estos mismos profesionales que se encuentren certificados y actualizados para aplicar su conocimiento y experiencia en los atletas; por ello es primordial de igual manera, formar equipos profesionales multidisciplinarios estratégicos que nos den logros en el ámbito del alto rendimiento, ávido de resultados a nivel internacional.

Enfocar los planes estratégicos a los dos pilares de nuestra sociedad que resultan más favorables para la educación y el fomento de hábitos de ejercicio: la escuela y la familia, en donde nuestros(as) jóvenes deben de tener el mayor apoyo.

Capacitar e involucrar a directivos, profesores de las diversas instituciones de educación, así como a los padres de familia sobre las estrategias a seguir para implementar actividad física en los escolares y en el núcleo familiar.

Realizar evaluaciones morfofuncionales a los escolares y universitarios para detectar problemas de salud, capacidades físicas, individualizar cargas de entrenamiento y formar grupos homogéneos para la aplicación de la actividad física en las escuelas. Formando grupos de profesionales para supervisar, evaluar y dar seguimiento a los programas 
establecidos, para ajustar o modificar en caso necesario. Así como elaborar informes periódicos sobre los avances obtenidos durante el desarrollo de los programas establecidos.

En el rubro de las evaluaciones morfofuncionales es sumamente crítico que la mayoría de nuestra población que realiza algún tipo de actividad deportiva, ni siquiera sepa que existen; pues estamos acostumbrados al famoso certificado médico general previo, como requisito para iniciar la práctica deportiva. Una evaluación morfofuncional debiera ser aplicada a toda aquella persona que realice ejercicio, y definitivamente va más allá de un simple certificado médico. La evaluación morfofuncional es una valoración cuantitativa y cualitativa de parámetros bioquímicos, psicológicos, mecánicos, antropométricos, energéticos y somáticos que determinan el rendimiento físico; basada en la realización de un examen clínico, exámenes de laboratorio, gabinete, antropometría, somatoscopía, pruebas funcionales y de esfuerzo. Todo esto para evitar entre otras cosas, lesiones que pueden llegar hasta la muerte súbita en el deporte.

Se debe evitar al máximo el estilo de vida sedentario, hay que enseñar y orientar a los y las jóvenes en el adecuado uso y manejo de las tecnologías a su alcance, evitando el abuso de las mismas, creando talleres y estrategias para la administración del tiempo libre; implementando programas académicos en educación física a nivel escolar, adecuando infraestructura apropiada al nivel y tipo de deporte, recursos materiales, y expertos en el área que apliquen sistemas efectivos y evaluables.

Por último, el introducir la educación física o la práctica deportiva como una actividad igual de importante que las académicas, en el nivel universitario y escolar, de forma organizada, supervisada y profesionalizada, ayudará a que los y las jóvenes aprecien y obtengan de manera precisa los beneficios que todo esto les otorga. 
URL www.una.ac.cr/mhsalud

\section{CONCLUSION}

Los y las jóvenes son el semillero de la fuerza laboral y cultural, y lo que suceda con ellos y ellas será nuestro futuro como país, por lo que habrá que cuidar esa base poblacional para que se pueda mantener un equilibrio. La obesidad, realidad crítica instalada en la población, principalmente producto de una vida moderna y apegada a la tecnología sobre todo en este grupo etáreo, debe ser abordada en base a estrategias y políticas públicas construidas pensando en la situación real que nos aqueja y pensando que el problema demanda una atención pronta, con soluciones eficaces que no pueden esperar a una resolución a largo plazo, se deben tomar medidas a corto plazo que puedan revertir la situación que se vive hasta este momento, pues de no ser así, nuestro país se encuentra en el peligro inminente de ser integrado por futuros adultos mayores repletos de enfermedades crónico degenerativas e incapacidades físicas que llevarán la economía y el equilibrio laboral a una situación deplorable.

El educar en la salud es un área determinante para poder transformar hábitos y costumbres de la sociedad que puedan resultar en una mejor calidad de vida, teniendo como una herramienta básica la promoción de la actividad física y el deporte a nivel nacional, ya que quedan completamente comprobados los beneficios en la salud que su práctica adecuada conlleva. Pero, es importante que se haga conciencia que esta actividad física va más allá de subir las escaleras en vez de usar un elevador por ejemplo, o de recomendar comer frutas y verduras diariamente; requiere de mucho más, de que cada individuo cuente con un plan de entrenamiento individual con metas y objetivos específicos y alcanzables, que conjuntamente se lleve a cabo con un régimen alimentario igualmente individualizado, dejando atrás los productos milagro y la "varita mágica" esperada por la mayoría para que nos resuelva la situación sin que ocupemos el mayor esfuerzo.

Así también se deben establecer las bases estrechando lazos con otras áreas de la salud como la Medicina del Deporte, para desarrollar programas y sistemas de activación física aplicables a nuestra población, ayudando de esta manera a mejorar el estilo de vida en lo 
individual y lo colectivo, no solamente en el mantenimiento de la salud, sino optimizando y aumentando el nivel de rendimiento físico en general. Todo esto, tomado en cuenta como principales áreas de oportunidad e intervención a pilar fundamental de la sociedad que es la familia, y al pilar de mayor influencia y formación en el país, que es la escuela.

Con respecto al comparativo entre las universidades públicas y privadas en los aspectos de la infraestructura y recursos humanos, cabe señalar, que la infraestructura en áreas deportivas se encuentra basta en la mayor parte de las instituciones privadas, así como la presencia del recurso humano especializado igualmente en la mayoría de ellas, sin embargo, un buen porcentaje de alumnos de esta población cuenta con cierta apatía a realizar una disciplina deportiva con regularidad y compromiso; así también aunque se cuenta con médicos en medicina del deporte y algunos otros profesionales en el área, son pocos en cantidad, para el número de eventos y actividades que se desarrollan en sus instalaciones.

En las universidades públicas en general, hace falta poner énfasis en la infraestructura y en los recursos humanos especializados para satisfacer la demanda del gran número de alumnos que manejan en sus filas. Aunque la población de estas universidades es más cooperadora y entusiasta, hace falta encaminar de manera adecuada esta actitud hacia una práctica deportiva equilibrada y eficaz.

De manera genérica en ambos grupos universitarios, hace falta inmiscuir profesionales de la medicina deportiva, nutrición, psicología y entrenamiento, que trabajando de manera interdisciplinaria puedan aportar conocimientos y experiencia profesional que de un giro de $360^{\circ}$ a la situación actual de la actividad física que vive el sector universitario en nuestro país. Y esto no sucederá hasta que los encargados de dirigir y administrar la educación en nuestro medio estén concientes de estas necesidades. 
URL www.una.ac.cr/mhsalud

\section{REFERENCIAS.}

Blanco, S. (2006). Las tecnologías de la información y comunicación: factores determinantes de los cambios culturales entre jóvenes usuarios. Razón y Palabra. Tecnológico de Monterrey Campus Irapuato.

Carratala, V.; Carratala, E. (1999). El judo en edades tempranas. Una propuesta de competición. Ponencia en el primer congreso de la actividad física y el deporte en la universidad. Universidad de Valencia. Facultad de Ciencias de la Actividad física y el deporte.

Devís, J.; Peiro, C. (1997). Nuevas perspectivas curriculares en Educación Física: la salud y los juegos modificados. Zaragoza, España.

Devís, J.; Peiro, C. (1992). Exercise and health in a Spanish physical education curriculum: A modified programme of "The exercise challenge". En T. Williams, L. Almond y A. Sparkes (eds.) Sport and physical activity: Moving towards excellence, Londres.

García, H. (2011). Nadie sabe a donde llegará la expansión de las RSD: expertos. En periódico La Jornada, México; 14-junio-2011. Recuperado desde: www.jornada.unam.mx/2011/06/14/sociedad/040nlsoc.

Gutiérrez, S. (1995). Valores sociales y deporte. Editorial Gymnos, Madrid, España.

Grandes, G.; Sánchez, A.; Torcal, J. (2003). Protocolo para la evaluación multicéntrica del programa experimental de promoción de la actividad física. Atención Primaria. 475-480.

Moya, S.; García, A.; Lucena, N.; Casañas, R.; Brito, P.; Rodríguez, A.; Flores, Z.; Cordero, R. (2006). Hipocinetismo: ¿un problema de salud en los jóvenes ucevistas? RFM.1 (29), junio, Caracas. 
Paffenbarger, R.S.; Hyde, R.T.; Wing, A.L.; Lee, I.M.; Jung, D.L.; Kampert, J.B. (1993). The association of changes in physical-activity level and other lifestyle characteristics with mortality among men. New England Journal of Medicine, 328, 538-545.

Pate, R. (1995). Physical activity and health: dose-response issues. Research Quarterly for Exercise and Sport, 66 (4): 313-317.

Quevedo, L. (2003). La escuela frente a los jóvenes, los medios de comunicación y los consumos culturales en el siglo XXI. Educación media para todos. UNESCOALTAMIRA-FUND. OSDE. Buenos Aires.

Ramírez, W.; Vinaccia, S.; Suárez, G. (2004). El impacto de la actividad física y el deporte sobre la salud, la cognición, la socialización, y el rendimiento académico: Una revisión teórica. Revista de Estudios Sociales, № 18, 67-75.

Rodríguez, F. (1995). Prescripción de ejercicio y actividad física en personas sanas (I). Principios generales. Atención Primaria, N¹5, 190-194.

Santos, S. (2005). La educación física escolar ante el problema de la obesidad y el sobrepeso. Revista Internacional de medicina y Ciencias de la Actividad Física y el Deporte, 5.

Simons-Morton, B.G.; Parcel, G. S. (1988). Health-related physical fitness in childhood: status and recommendations. Annual Review of Public Health, $\mathrm{N}^{\circ}$ 9, 403-425.

Varo Cenarruzabeitia, J.J; Martínez Hernández, J.A.; Martínez González, M.A. (2003). Beneficios de la actividad física y riesgos del sedentarismo. Medicina Clínica. Madrid España. 
URL www.una.ac.cr/mhsalud

WHO. (1998). Obesity: Preventing and Managing. The Global Epidemic. Report of a WHO Consultation on Obesity. Geneva.

Fecha de recepción: 22 de junio de 2011.

Fecha de aceptación: 9 de noviembre 2012.

Fecha de publicación: 31 de diciembre del 2012. 